\title{
MAPPING CYLINDER NEIGHBORHOODS OF SOME ANR'S
}

\author{
BY R. T. MILLER ${ }^{1}$ \\ Communicated October 2, 1974
}

Let $X$ be a closed subset of the interior of a manifold $Q$. A submanifold $M$ of $Q$ is a mapping cylinder neighborhood of $X$ if it is a closed neighborhood of $X$ in $Q$ and if there is a proper map $r: \partial M \rightarrow X$ such that $M$ is homeomorphic to the mapping cylinder of $r$, fixing $\partial M$ and $X$ in the natural way. Since mapping cylinders strong deformation retract to their targets, a set that possesses a mapping cylinder neighborhood in some manifold is a finite dimensional ANR. Moreover, if the set is compact, its mapping cylinder neighborhood is a compact manifold. It follows from Kirby and Siebenmann $[\mathbf{K}-\mathbf{S}]$ that the neighborhood, hence the set, has finite homotopy type.

Regular neighborhoods of locally finite complexes in $P L$ manifolds are mapping cylinder neighborhoods. More generally, R. D. Edwards showed [E] that stably, locally finite cell complexes in manifolds have mapping cylinder neighborhoods. We prove the following

THEOREM. Let $M^{\prime}$ be a manifold. Suppose $M=M^{\prime} \cup$ (open outside collar of $\left.\partial M^{\prime}\right)$, and that $M$ supports a fixed point free flow whose flow lines give an oriented foliation on $M$ that is transverse and outward pointing on $\partial M^{\prime}$. Suppose $X$ is a locally compact ANR embedded as a closed, $1-L C$, codimension 4 subset of the interior of a manifold $Q$. Then $X \times M^{\prime}$ has a mapping cylinder neighborhood in $Q \times M$.

We recover as a corollary the result of Gersten [G] and Wall [W] that the product of a compact, finite dimensional ANR with a compact manifold of Euler characteristic zero has finite homotopy type.

More significant is the case where $M^{\prime}=(-1,1]$ and $M=(-1, \infty)$. It suggests the possibility of joining two copies of a mapping cylinder neighborhood of $X \times(-1,1]$ back to back in order to get a mapping cylinder neighborhood of $X \times[-1,1]$ in some manifold, which easily implies the

AMS (MOS) subject classifications (1970). Primary 54C55, 57A40.

${ }^{1}$ Alfred P. Sloan Fellow. This work was done at the Institut des Hautes Études Scientifique. 
existence of a mapping cylinder neighborhood for $X$ itself in that manifold. J. E. West has been able to carry out a somewhat more complex program in Hilbert Cube manifolds, to prove that compact ANR's have finite homotopy type. (See subsequent announcement, this Bulletin.) More recently, Edwards and L. C. Siebenmann have used West's idea and a finite dimensional version of his principal tool to carry out directly the joining indicated above.

\section{REFERENCES}

E. R. D. Edwards, Topological regular neighborhoods (to appear).

G. S. M. Gersten, A product formula for Wall's obstruction, Amer. J. Math. 88 (1966), 337-346. MR 33 \#6623.

K-S. R. C. Kirby and L. C. Siebenmann, On the triangulation of manifolds and the Hauptvermutung, Bull. Amer. Math. Soc. 75 (1969), 742-749. MR 39 \#3500.

W. C. T. C. Wall, Finiteness conditions for CW complexes, Ann. of Math. (2) 81 (1965), 56-69. MR 30 \#1515.

DEPARTMENT OF MATHEMATICS, MICHIGAN STATE UNIVERSITY, EAST LANSING, MICHIGAN 48823

Current address: Department of Mathematics, University of California, Berkeley, California 94720 